\title{
Experimental observation of Dyakonov plasmons in the mid-infrared
}

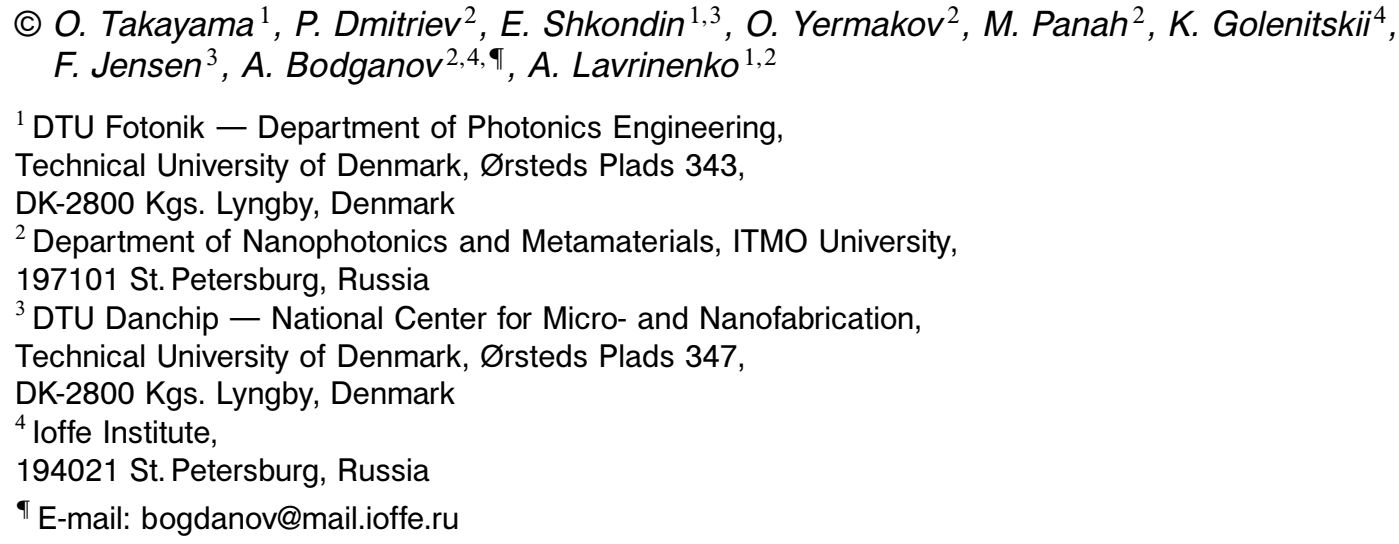

In this work,we report on observation of Dyakonov plasmons at an interface with a hyperbolic metamaterial in the mid-IR.The hyperbolic metamaterial is implemented as a CMOS-compatible high aspect ratio grating structure with aluminium-doped $\mathrm{ZnO}(\mathrm{AZO})$ ridges grown by atomic layer deposition in deep trench silicon matrix.The dispersion of Dyakonov plasmons is characterized by the attenuated total reflection method in the Otto configuration. We demonstrate that Dyakonov plasmons propagate in a broad range of directions (a few tens of degrees) in contrast to the classical Dyakonov surface waves (about one tenth of degree).The obtained results provide useful guidelines for practical implementations of structures supporting Dyakonov plasmons in the mid-IR.

Acknowledgment This work was supported by VillumFonden DarkSILD" project No.11116, the Russian Foundation for Basic Research (16-37-60064, 17-02-01234), the Ministry of Education and Science of the Russian Federation (3.1668.2017/4.6), the President of Russian Federation (MK-403.2018.2). 